\title{
Photometry of red giants in the disk of IC 1613
}

\author{
N. A. Tikhonov ${ }^{1,2}$ and O. A. Galazutdinova ${ }^{1,2}$ \\ 1 Special Astrophysical Observatory, Russian Academy of Sciences, N. Arkhyz, KChR 369167, Russia \\ 2 Isaac Newton Institute of Chile, SAO Branch, Russia
}

Received 27 September 1999 / Accepted 30 May 2002

\begin{abstract}
The results of photometry of disk stars in the galaxy IC 1613 are presented. The position of the red giant branch allowed us to measure the distance to the galaxy $(m-M)=24.53 \pm 0.10$, as well as metallicity $([\mathrm{Fe} / \mathrm{H}]=-1.75)$ and age $(\lg t \simeq 10.1)$ of disk stars. The variation of the surface density of red giants depending on the distance from the center of the galaxy has been found. Rough estimate of the total number of red giants in the galaxy and total disk mass are presented.
\end{abstract}

Key words. galaxies: individual: IC 1613 - galaxies: photometry - galaxies: stellar content

\section{Introduction}

The irregular galaxy IC 1613, being a member of the Local group, was the subject of costant interest for astronomers for a few decades. The close position of this galaxy makes it possible to study in detail the stellar population in it. Cepheids, which were observed in IC 1613 since the time of Baade, allowed one to obtain quite reliable estimates of the distance to this galaxy (Baade 1963; Sandage 1971; van den Bergh 1975; van den Bergh 1977; de Vaucouleurs 1978; Mc Alary et al. 1984; Freedman 1988a; Madore \& Freedman 1991; Carlson \& Sandage 1990; Dolphin et al. 2001). Using a different method (TRGB method) based on the observations of red giants (Freedman 1988b; Cole et al. 1999) determined the distance to IC 1613. The third method of distance estimation to this galaxy appeared in the work of Saha et al. (1992) who revealed RR Lyr-type stars in IC 1613 on deep CCD images.

A comparatively small region $(16.2 \times 14.5$ from $\mathrm{RG} 3)$ of the visible part of the galaxy permits investigators to measure easily its global characteristics. The total magnitude of the galaxy, $B_{t}=9$ m. 88 , corresponds to $M_{b}=-14^{\mathrm{m}} .5$ with distance modulus $(m-M)=24$. 4 and its colour index increases from the centre towards the edge (Hodge 1978); the mean value $(B-V)=0.67$ (RC3).

The process of star formation in the galaxy is suggested by the presence of a system of HII regions in it (Sandage 1971; Lequeux et al. 1987; Price et al. 1990; Hodge et al. 1990) and is confirmed by the discovery of numerous young stars there (Baade 1963; Sandage 1971; Freedman 1988b; Hodge 1978; Hodge et al. 1991; Armandroff \& Massey 1985; Elias \& Frogel 1985; Georgiev et al. 1999; Cole et al. 1999). Observations in HI have shown a large amount of hydrogen to be present with the maximum concentration coinciding with star formation

Send offprint requests to: N. A. Tikhonov, e-mail: ntik@sao.ru regions; the effective size of the galaxy in HI equals $24^{\prime}$, which corresponds to the disk size of red giants from Baade's measurements (Volders \& Hogbom 1961; Rots 1980; Huchtmeier et al. 1981; Lake \& Skilman 1989; Lu et al. 1993; Hoffman et al. 1996). The maximum diameter of the galaxy in neutral hydrogen is essentially larger and equals 54' (Hoffman et al. 1996).

It can be seen from the results presented above that the galaxy IC 1613 was actively studied in past and now is used as a standard for this type of irregular galaxy. However almost all previous results are connected with the central regions of IC 1613, paying no attention to the outer parts of the disk, where there is some important information about the early stage of the galaxy formation.

In our paper we present the photometric results for very old disk red giants, their surface distribution along the galaxy radius and the estimates of their ages and metallicities.

\section{Observation and reduction}

CCD images of a part of the IC 1613 disk were obtained with the 6-m telescope BTA in the $R$ and $I$ filters on January 19, 1999 (Figs. 1 and 2). The position of the images was selected at the edge of the disk at a distance of $10^{\prime}$ east of the centre. The need for this displacement is explained by the fact that bright stars (supergiants) are abundant in the central regions and so decrease the accuracy of photometry of neighboring faint stars - red giants in our case. Also, in this study we are trying to detect the boundary of the galactic disk consisting of old stars. The disk boundary can be revealed by an abrupt decrease in the number of red giants and is clearly seen in the spiral galaxy M 33 (Cuillandre et al. 1998). We found a quite similar manifestation of the disk boundary in the irregular galaxy IC 10, which resembles IC 1613 and is placed at nearly 


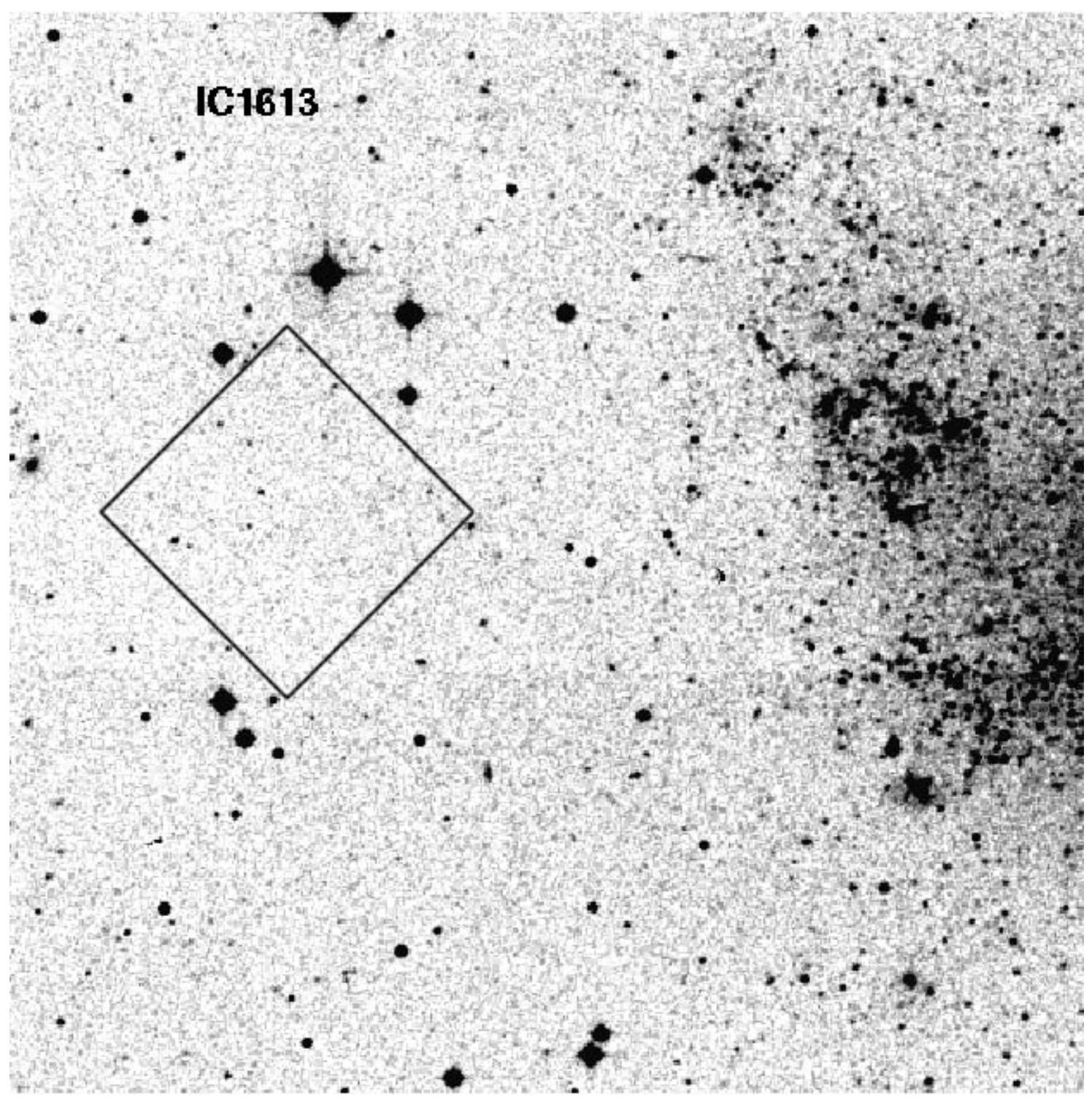

Fig. 1. The reproduction from Digital POSS-1 of the part of IC 1613 with the marking of the 6-m telescope field.

the same distance from the Galaxy but suffers strong light absorption by the Milky Way (Tikhonov 1998). The conditions of the observations with the 6-m telescope are listed in Table 1. We used a "Photometrics" CCD with $1024 \times 1024$ pixels and a scale of $0.214^{\prime \prime} /$ pix. All the images were subjected to a standard procedure of reduction (bias, flat field, cosmic). The photometry of stars on all images was performed with the DAOPHOT II software package (Stetson 1993). The transformation equations from the instrumental values to the RI system (Kron-Cousins) were obtained from photometry of the standard stars from the list of Landolt (1992), which were observed over the whole night.

\section{CMD, the distance and metal abundance}

Figure 3 shows the CM diagram for the disk stars we have observed. The main feature of the diagram is the presence of the well-distinguished red giant branch with a small number of asymptotic giant branch stars. The two comparatively bright
Table 1. Log of observations.

\begin{tabular}{ccrcc}
\hline \hline Date & Filter & $T_{\exp }$ & A.Mass & Seeing \\
\hline 18.01 .99 & $R$ & 30 & 1.45 & 1.2 \\
18.01 .99 & $R$ & 600 & 1.46 & 1.3 \\
18.01 .99 & $I$ & 100 & 1.51 & 1.1 \\
18.01 .99 & $I$ & 600 & 1.51 & 1.1 \\
\hline
\end{tabular}

stars (not presented on the CM diagram) were defined by us as background stars on the basis of the measurement of their colour and position on the diagram. There are only few faint background stars on the CM diagram, and they do not affect TRGB or $[\mathrm{Fe} / \mathrm{H}]$ measurements. One can see a lot of diffuse objects on the images presented which are in fact distant galaxies: their nature is obvious from the morphology of the brightest of them. We have failed to find stellar clusters in the images, but this is quite natural since the region under investigation is 


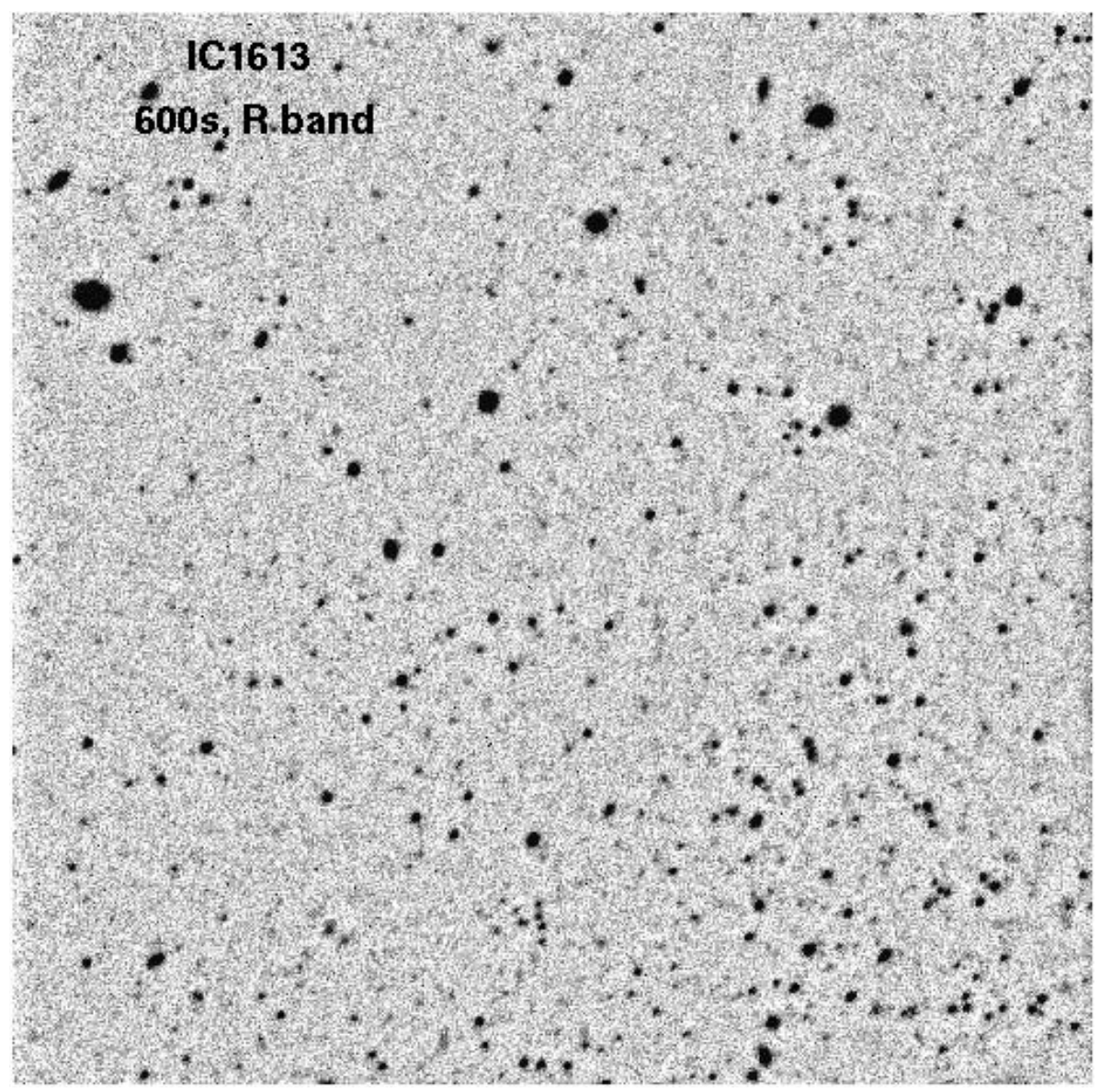

Fig. 2. R-image of the part of the disk of IC 1613. For this $3.5 \times 3.5$ frame, north is to the top-right corner of the image.

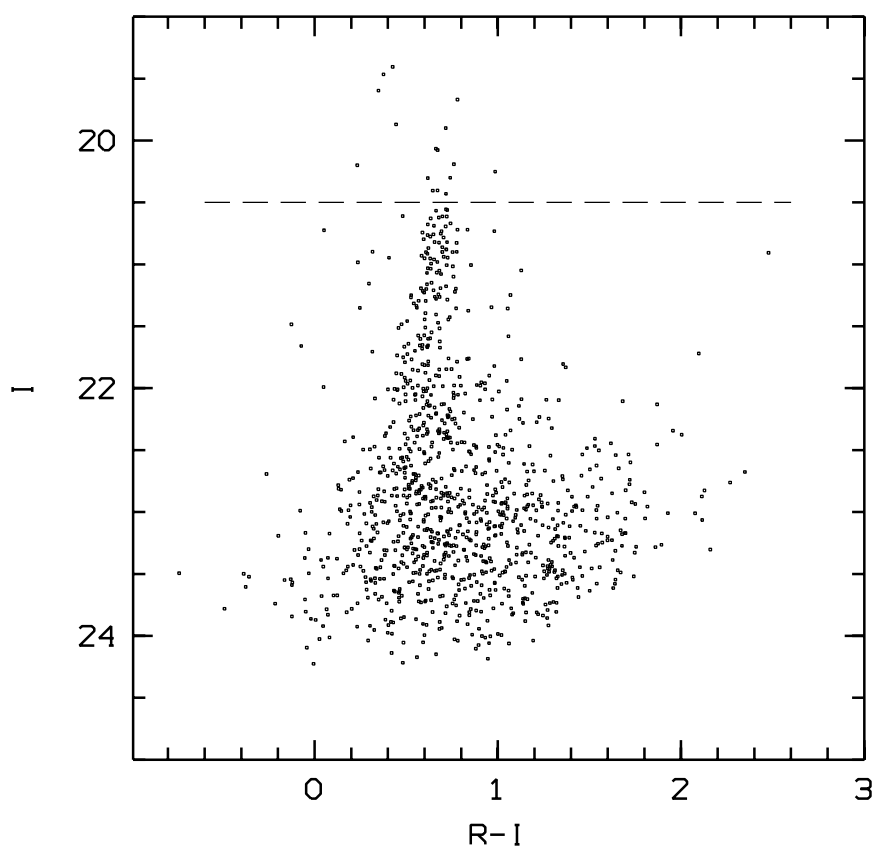

Fig. 3. Observational $I$ vs. $(R-I)$ CM diagram of the part of IC 1613 . The dashed line shows the position of the TRGB.

located at the edge of the galaxy. Thus we only observe the giants alone on the CMD. The comparatively large width of the red giant branch can partly be explained by the photometric errors given in Figs. $4 \mathrm{a}$ and $4 \mathrm{~b}$ and in part by differences in the metal abundances. Completeness tests were performed using the usual procedure of artificial star trials (Stetson 1987). 6000 artificial stars were added to the $R$ frame of IC 1613 in several steps of 600 stars each with magnitudes in the range of $21 \leq R \leq 25$. The completeness curves (input magnitudes fraction recovered) are shown in Fig. 5. It is worth noting that some Local group galaxies have even wider red giant branches on the CM diagram. For IC 1613 the red giant branch (RGB), which is easily separated from the asymptotic giant branch (AGB), cuts off at $I=20.55 \pm 0.05$ (Fig. 6). From the results of Schlegel et al. (1998) we have for IC $1613 E(B-V)=0.025$ and $A_{I}=0.049, E(R-I)=0.018$. The internal reddening in our region is very negligible, because of the long distance from star formation regions.

By applying the TRGB method developed by Lee et al. (1993) we find the distance from the galaxy and the metallicity of red giants. From the $I$-band luminosity function shown in Fig. 6, we have $I_{\mathrm{TRGB}}=20.55$ and $(R-I)_{-3.5}=0.67 \pm 0.10$ that corresponds to $(V-I)_{-3.5}=1.35$. We converted $(R-I)$ to $(V-I)$ by Porter's data for global clusters M 67, M 92, NGC 2264, NGC 2419, NGC 4147, NGC 7006 and NGC 7790. The transformation equation is:

$V-I=1.91 \times(R-I)+0.054, \quad \sigma=0.06$. 


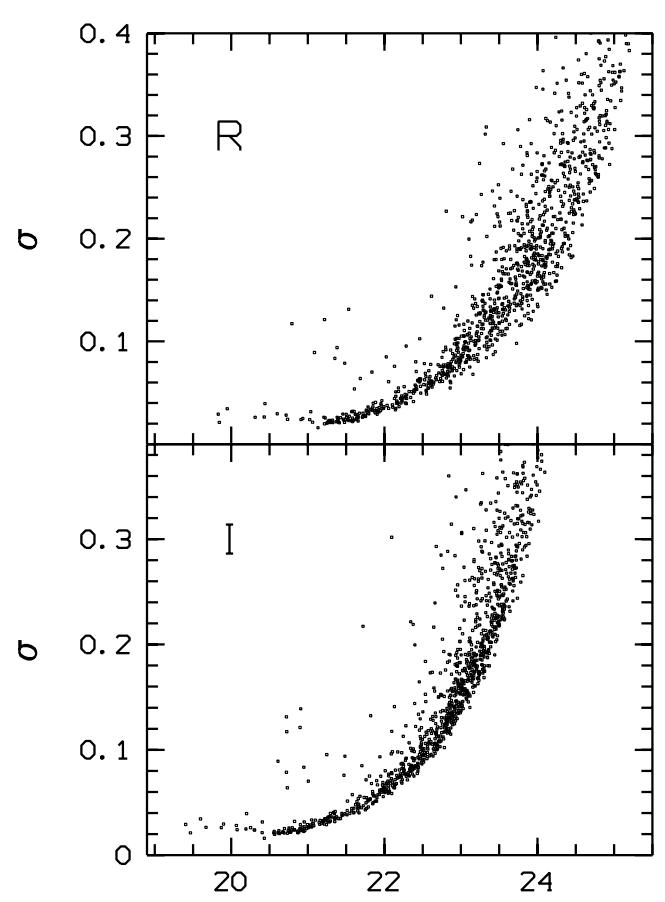

Fig. 4. The estmated standard errors at variance of $R$ and $I$ magnitudes as given by DAOPHOT II.

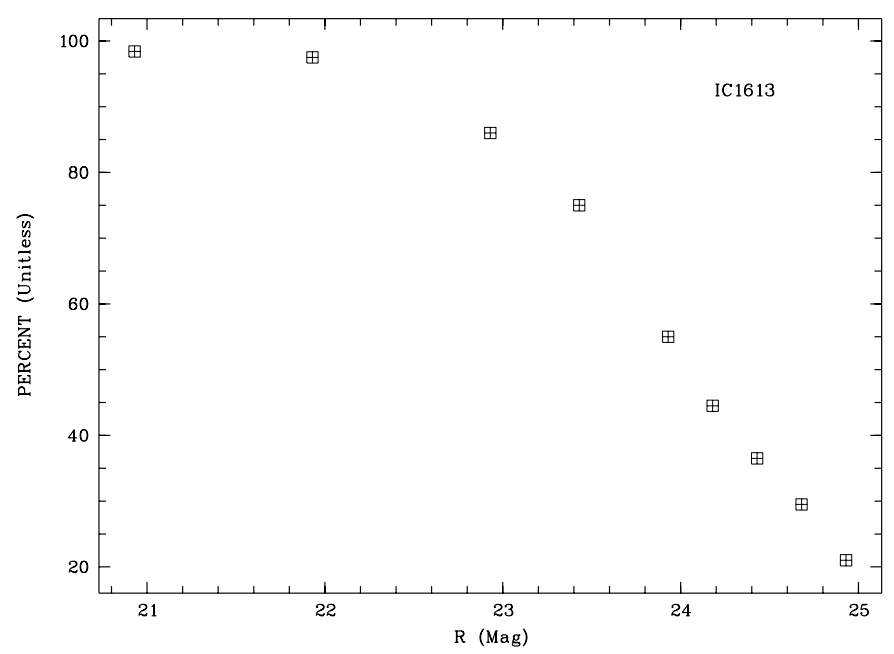

Fig. 5. Completeness fraction as a function of $R$ magnitude.

Then we find: $[\mathrm{Fe} / \mathrm{H}]=-12.64+12.6(V-I)_{0,-3.5}-3.3(V-$ $I)_{0,-3.5}^{2}=-1.75, B C_{I}=0.881-0.243(V-I)_{0,-3.5}=0.56$, $M_{\mathrm{bol}}=-0.19[\mathrm{Fe} / \mathrm{H}]-3.81=-3.48, M_{I}=M_{\mathrm{bol}}-B C_{I}=-4.03$, $(m-M)=20.55-0.049+4.03=24.53$. Thus $(m-M)=$ $24.53 \pm 0.2$, which corresponds to the distance of $805 \pm 80 \mathrm{kpc}$.

We can compare our results with those obtained by other authors from the Table 2, where the values of distances from IC 1613 are given.

The short TRGB scale distance of Freedman (1988b) may be the result of the crowding in the central part of the galaxy. This effect was studied by Madore \& Freedman (1995). Our estimate of the distance is subject to effect of the population size (Salaris \& Cassisi 1997), that can result in an overestimate the apperent distance modulus up to 0.15

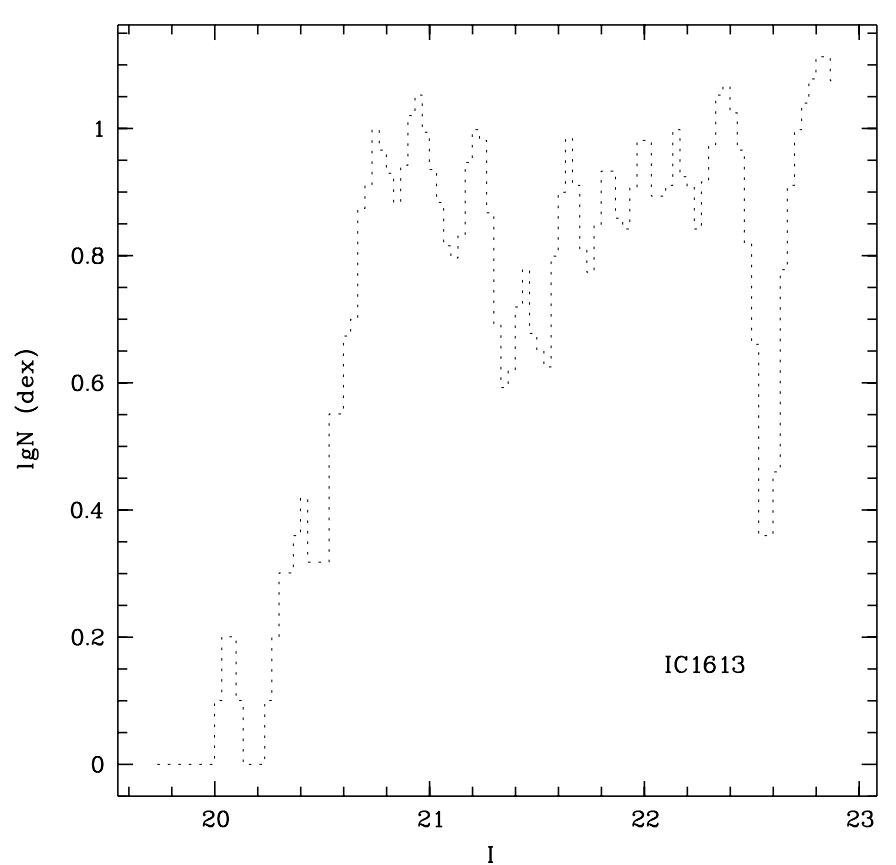

Fig. 6. RGB luminosity function. The bins are 0.03 wide. The tip of the RGB is at $I=20.55 \pm 0.05$.

Table 2. The distance to IC 1613 by different methods.

\begin{tabular}{lccccc}
\hline \hline Authors & Year & Method & $E(B-V)$ & $(m-M)$ & Dist. \\
\hline Sandage & 1971 & Cepheids & 0.04 & 24.43 & 770 \\
van den Bergh & 1975 & Cepheids & 0.03 & 24.73 & 880 \\
van den Bergh & 1977 & Cepheids & 0.03 & 24.41 & 760 \\
de Vaucouleurs & 1978 & Cepheids & 0.05 & 24.02 & 640 \\
Mc Alary et.al. & 1984 & Ceph. IR & & $24.31 \pm 0.12$ & 730 \\
Freedman & $1988 \mathrm{a}$ & Cepheids & 0.03 & $24.30 \pm 0.1$ & 725 \\
Freedman & $1988 \mathrm{~b}$ & TRGB & 0.03 & $24.20 \pm 0.2$ & 690 \\
Saha et al. & 1992 & RR Lyr & 0.02 & $24.10 \pm 0.27$ & 660 \\
Cole et al. & 1999 & TRGB & 0.03 & $24.29 \pm 0.1$ & 715 \\
Macri et al. & 2001 & Cepheids & & $24.44 \pm 0.13$ & 773 \\
Dolphin et al. & 2001 & Cepheids & 0.02 & $24.31 \pm 0.07$ & 728 \\
This paper & & TRGB & 0.02 & $24.53 \pm 0.10$ & 824 \\
\hline
\end{tabular}

(Madore \& Freedman 1995). The TRGB data with HST high space resolution are within our measurement errors.

The metallicity value for giants we obtained, $[\mathrm{Fe} / \mathrm{H}]=$ $-1.75 \pm 0.20$, reveals a low abundance of metals, which can easily be seen on the CM diagram from the giant branch position. The low content of metals is confirmed also by the isochrones of Berttelli et al. (1994) presented on the CM diagram. Among all isochrones, only that with the lowest content of metals $(z=0.0004$ and $z=0.001)$ describes the giant branch behaviour well (Fig. 7). The metallicity value found from the RGB position are as follows: $[\mathrm{Fe} / \mathrm{H}]=-1.3 \pm 0.8$ (Freedman 1988b), $[\mathrm{Fe} / \mathrm{H}]=-1.3 \pm 0.2$ (Dolphin et al. 2001) and $[\mathrm{Fe} / \mathrm{H}]=-1.38 \pm 0.31$ (Cole et al. 1999). Our metallicity estimate coincides with the results of other authors within the measurement errors. 


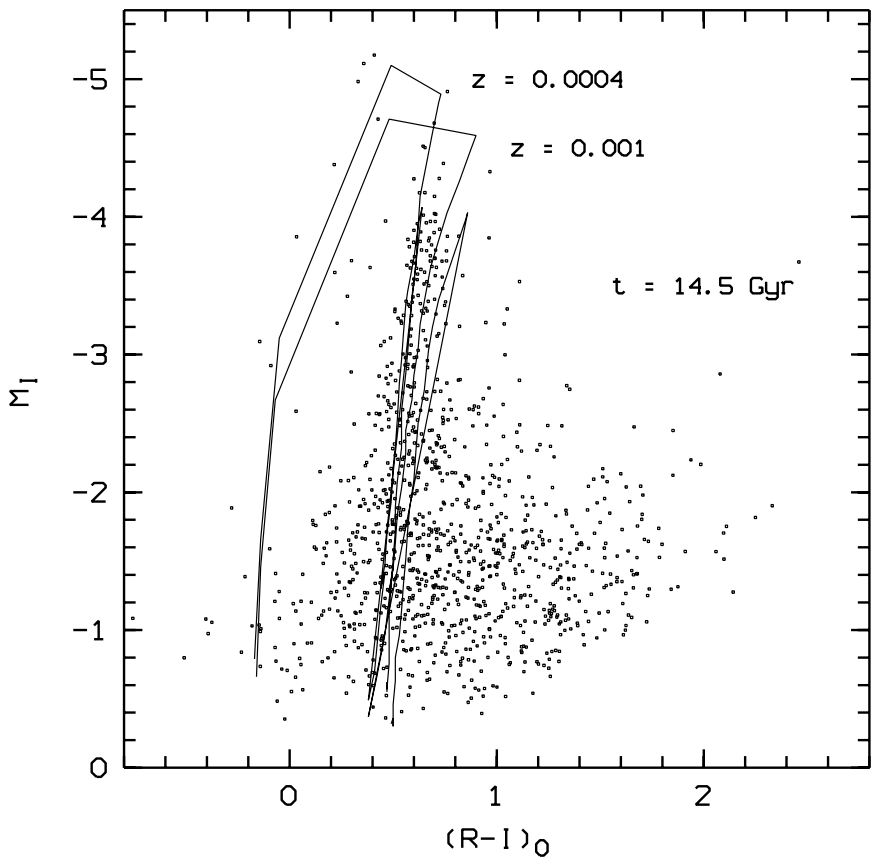

Fig. 7. The Padova isochrones for metallicity $z=0.0004$ and $z=$ 0.001 (Bertelli et al. 1994) with the CMD for the disk of IC 1613.

\section{The age of RGB stars}

The limiting stellar magnitude on the CM diagram we have obtained is $R=25$, which is not enough to apply usual methods of measuring the star ages. However, the use of theoretical isochrones allows one to estimate (at least roughly) the age of the stellar population under investigation. By selecting isohrones of different ages we have found that only the isochrones with $\lg t \simeq 10.1$ ( $t \simeq 14 \mathrm{Gyr}$ ) can explain well the structure of the CM diagram. Any lower value of $t$ requires the presence of stars brighter than those we see on our CM diagram. The size of our area $(800 \times 800 \mathrm{pc}$ for the distance value of IC 1613 we have found) permits us to be certain that the absence of younger giants cannot be explained by the small statistical sample in our measurements, but most likely reflects the process of instant star formation which happened about $14 \mathrm{Gyr}$ ago. Such a high value for the age of disk stars we have obtained here is not unexpected since for example the spheroidal galaxy Draco Dwarf is composed of similar old population II stars (Grillmair et al. 1998). We also see quite a similar CM diagram for the dwarf galaxy Tucana, which has an old stellar population and a low metallicity (Castellani et al. 1996). The discovery the RR Lyr stars in IC 1613 at $11^{\prime}$ from the galaxy center (Dolphin et al. 2001) has confirmed the existence of an old stellar population in the disk.

\section{Radial distribution of RGB stars}

By dividing the image into separate regions we have found the variation of the number of stars with the distance from the galactic centre. In the $R$-band we can reveal more faint objects than in the $I$-band, therefore, to obtain better statistical results, we used only $R$-band stars. We believe this procedure to be correct since nearly all faint stars on the CM diagram are red

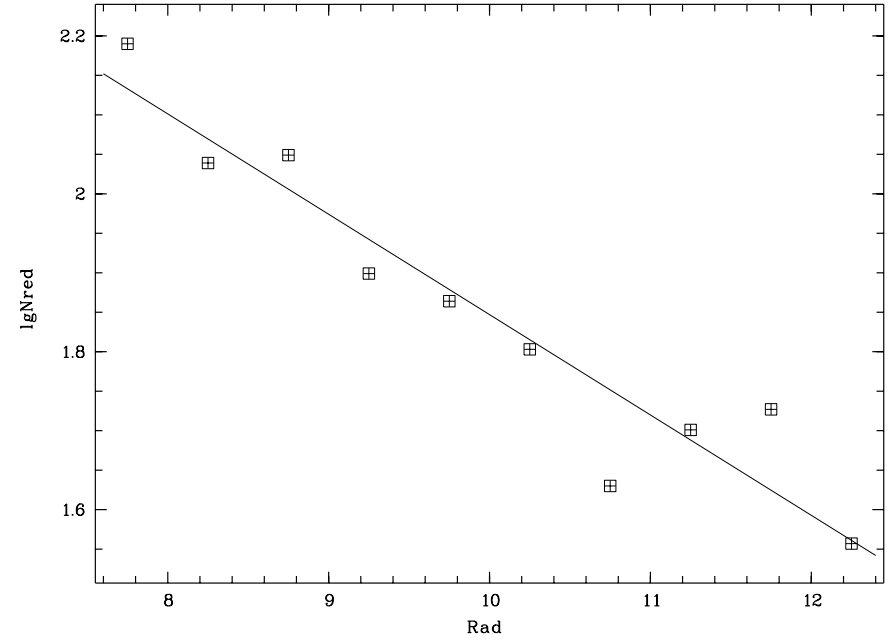

Fig. 8. The variation of the number of red giants with the distance from the center of the galaxy.

giants. The variation of the number of giants with the distance from the center of the galaxy that we have obtained is presented in Fig. 8. One can see that this variation has an exponential character, i.e. giants belong to the disk population. A similar behaviour of red giants was observed in all irregular galaxies for which we had deep CM diagrams. The detailed discussion of this question is presented elsewhere (Tikhonov 2001).

The smooth variation of the number of giants with radius up to the distance of about $12^{\prime}$ from the center of the galaxy suggests that we have not yet reached the edge of the disk. Our investigations of the HST archive images (PEP ID 5450, 6675 ) situated at $27^{\prime}$ and $33^{\prime}$ from the galaxy centre have not confirmed the existence of an old stellar population at these galactocentric radii.

Using the results of our analysis for the distribution of red giants with radius and assuming the galaxy disk size proposed by Baade (1963), one can find the total number of red giants in the galaxy and try to estimate the total mass of the disk. We will base our estimates on the results obtained for the galaxy Draco, where deep photometry of stars is available (Grillmair et al. 1998). One has to accept the assumption that the stellar population is homogeneous throughout the disk and that the evolution of stars in Draco and in the disk of IC 1613 are similar. The latter assumption follows from the similarities in the metallicities and ages of the red giants in these two galaxies.

Assuming for the disk size of IC 1613 the value 25' (Baade 1963 ) we obtain the total number of giants $N=2.3 \times 10^{4}$. After the correction for the completeness of the sample (Fig. 5), this number increases: $4.1 \times 10^{4}$. The second correction is connected to the low-mass stars, invisible in the case of IC 1613, but existing on the HST images of Draco we have mentioned above. For the Draco dwarf, the ratio of the number of red giants located above the horizontal branch to the total number of observed low-mass stars $N_{\text {red }} / N_{\text {tot }}$, equals 440 . Assuming the same ratio for the disk of IC 1613, we find that the total number of stars with masses larger than $0.8 M_{\odot}$ in the disk is $1.8 \times 10^{7}$. Considering the mean mass of the disk star to be that of the Sun, the final estimate for the mass of disk stars then is $1.8 \times 10^{7} M_{\odot}$. 
Since the luminosity $L$ of the whole galaxy IC 1613 is equal to $1.1 \times 10^{8} L_{\odot}$, the $M_{\text {disk }} / L_{\text {gal }}$ relation is 0.16 . This value is very small and it is possible that the main mass of the galaxy belong to the stars with the masses $<0.8 M_{\odot}$ assuming that the main mass of the galaxy is contained in the disk.

\section{Summary}

On the basis of the photometry of red giant for the irregular galaxy IC 1613 we have obtained metallicities and ages of disk stars and the distance from this galaxy. Comparison of the properties of the disk stars in IC 1613 with stars in the spheroidal dwarf galaxies Draco and Tucana shows their likeness within the measurement errors, both in age and metal content. An attempt has been made to estimate the number of stars in the disk and to determine its mass. The obtained value of the ratio $M / L$ is its lower limit because the contribution of low-mass stars could be underestimated.

Acknowledgements. We gratefully acknowledge Dr. E. Volkov for his helpful discussions. This work has been supported by RFBR, grant 00-02-16584.

\section{References}

Armandroff, T. E., \& Massey, P. 1985, ApJ, 291, 685

Baade 1963, in Evolution of Stars and Galaxies (Harvard University, Cambridge, MA)

Bertelli, G., Bressan, A., Fagotto, F., Choisi, C., \& Nasi, E. 1994, A\&AS, 106, 137

Carlson, G., \& Sandage, A. 1990, ApJ, 352, 587

Castellani, M., Marconi, G., \& Buonanno, R. 1996, A\&A, 310, 715

Cuillandre, J. C., Lequeux, J., \& Loinard, L. 1998, IAU Symp., 192

Cole, A. A., Tolstoy, E., Gallagher, J. C., et al. 1999, AJ, 118, 1657

Dolphin, A. E., Saha, A., Skillmann, E. D., et al. 2001, ApJ, 550, 554 de Vaucouleurs 1978, ApJ, 223, 730

Elias, J. H., \& Frogel, J. A. 1985, ApJ, 289, 141

Freedman, W. L. 1988a, ApJ, 326, 691
Freedman, W. L. 1988b, AJ, 96, 1248

Hodge, P. W. 1978, ApJS, 37, 145

Hodge, P. W., Lee, M. G., \& Gurwell, M. 1990, PASP, 102, 1245

Hodge, P. W., Smith, T. R., Eskridge, P. B., et al. 1991, ApJ, 369, 372

Hoffman, G. L., Salpeter, E. E., Farhat, B., et al. 1996, ApJS, 105, 269

Huchtmeier, W. K., Seiradaks, J. H., \& Materne, J. 1981, A\&A, 102, 134

Georgiev, L., Borissova, J., Rosado, M., et al. 1999, A\&AS, 134, 21

Grillmair, C. J., Mould, J. R., Holtzman, J. A., et al. 1998, AJ, 115, 144

Lake, G., \& Skilman, E. D. 1989, AJ, 98, 1274

Landolt, A. U. 1992, AJ, 104, 340

Lee, M. G., Freedman, W. L., \& Madore, B. F. 1993, ApJ, 417, 553

Lequeux, J., Meyssonnier, N., \& Azzopardi, M. 1987, A\&AS, 67, 169

Lu, N. Y., Hoffman, G. L., Groff, T., Roos, T., \& Lamphier, C. 1993, ApJS, 88, 383

Macri, L. M., Calzetti, D., Freedman, W. L., et al. 2001, ApJ, 549, 721

Madore, B. F., \& Freedman, W. L. 1991, PASP, 103, 933

Madore, B. F., \& Freedman, W. L. 1995, AJ, 109, 1645

Mc Alary, C. W., Madore, B. F., \& Davis, L. E. 1984, ApJ, 276, 487

Porter, A. C., unpublished data

Price, J. C., Mason, S. F., \& Gullixon, C. A. 1990, AJ, 100, 420

Rots, A. H. 1980, A\&AS, 41, 189

Saha, A., Freedman, W. L., Hoessel, J. G., \& Mossman, A. E. 1992, AJ, 104, 1072

Salaris, M., \& Cassisi, S. 1997, MNRAS, 289, 406

Sandage 1971, ApJ, 166, 13

Schlegel, D. J., Finkbeiner, D. P., \& Davis, M. 1998 ApJ, 500, 525

Stetson, P. B. 1987, PASP, 99, 191

Stetson, P. B. 1993, Users manual for DAOPHOT II

Tikhonov, N. A. 1998, IAU Symp., 192

Tikhonov, N. A. 2001, Astronomical Conference in St. Petersburg, August 6-12, 174

van den Bergh, S. 1975, in Galaxies and the Universe, Stars and Stellar Systems, Vol. IX, ed. A. Sandage, M. Sandage, \& J. Kristian (University of Chicago, Chicago), 518

van den Bergh, S. 1977, in Déclages vers le Rouge et Expansion de l'Univers, ed. C. Balkowski, \& B. E. Westerlund (CNRS, Paris), IAU Coll., 37, 13

Volders, L., \& Hogbom, J. A. 1961, BAN, 15, 307 